\title{
PIV measurements of entrainment process of directly injected media in internal combustion engines
}

\author{
Elsayed Abdelhameed ${ }^{1}$, Takahide Aoyagi ${ }^{1}$, Daisuke Tsuru $^{1}$, Hiroshi Tashima ${ }^{1 *}$ \\ ${ }^{1}$ Interdisciplinary Graduate School of Engineering Sciences, Kyushu University, Japan \\ *Corresponding Author: Hiroshi Tashima, Interdisciplinary Graduate School of Engineering \\ Sciences, Kyushu University 6-1 Kasuga-koen, Kasuga, Fukuoka, Japan Tel.: +81-92-583-7592 \\ Email: tasima@ence.kyushu-u.ac.jp
}

Abstract

PIV measurements have been successfully applied to various flow fields relating internal combustion engines such as in-cylinder air motion, air flow in an intake port, and even a discharging passage of an ignition plug. Measurements of induced air motion around diesel sprays can be said to be a significant example of the PIV applications because the air motion is reflected in an unsteady complicated flow structure. Instead of the apparent entrainment exaggerated by spray droplet dispersing, substantial air entrainment through momentum exchange between liquid and gas was finally obtained by combining PIV and spray profile observation.

PIV measurements of this kind were extensionally applied to other direct fluid injection by the authors. The second object was a high-pressure gas jet directly injected under gas pressure as high as $30 \mathrm{MPa}$. It was found the gas jet has strong air entrainment through momentum exchange in a single gaseous phase between fuel gas and ambient air. The third directly injected medium in internal combustion engines should be torch flame ejected from nozzle holes of a pre-combustion chamber (PCC) to a main combustion chamber (MCC) of a so-called DF (dual-fuel) engine.

In this study, mixture entrainment process of torch flames is discussed on the PIV results for the first time. However, chamber configurations of a real DF engine are hard to simulate since it requires several auxiliary PCC devices such as an ignition plug, a sub gas injector, and so on. All of them should be actuated synchronously with an engine crank angle. In the case of a constant volume vessel (CVV), the synchronization is not necessary, but the mixture control in the PCC becomes problematic because of the lack of compression and expansion strokes that assures PCC gas exchange. For overcoming the situation, rupture of a membrane was introduced in this study. The membrane turns the upper part of the PCC into an air pressure reservoir and low-pressure air jets eject from the nozzle holes after a solenoid-driven needle pierces the membrane for rupturing. The differential pressure between the upper chamber and the lower one was chosen as a main parameter of the experiment.

Since the measurements and analysis of the entrainment of the low-pressure air jets are yet to finalize, the outlook of the CVV, the PIV specifications, and prime results of the air entrainment 
are attached herewith. After all, the PIV measurements revealed essential difference among air entrainment processes of the above three directly injected media in internal combustion engines.

\section{Introduction}

Because of the high restrictions regulations to the emissions, the researchers payed intensive attention to investigate the combustion process aiming to enhance the fuel combustion process and reduce emissions Zhao et al. (2020). The in-cylinder combustion process is highly dependent on the fuel-air mixture as air entrainment phenomena Thiripuvanam et al. (2017). Not only burning diesel fuel, but large internal combustion engines that burn natural gas have also appeared in marine engines as well Healy et al., (2008); Lion et al. (2020). Therefore, studying the air entrainment for different fuel types can increase the combustion process knowledge. PIV technique has been used previously by many researchers. Xia et al. Xia et al. (2019) have studied in details the atomization of diesel spray in marine diesel scale concluding that the liquid phase of the spray is smooth and stable compared showing shorter penetration. Increasing the ambient pressure leads to decrease in tip velocity. Zhang et al. Zhang et al. (2019) used three different techniques to study the spray penetration and cone angles in marine diesel scale. The results showed that the higher the ambient temperature the lower the liquid penetration. The cone angle is highly affected by the ambient temperature and ambient density. PIV (Particle Image Velocimetry) is a general term for flow velocity measurement methods that identify the flow velocity based on the processing of particle images Zegers et al. (2012).

In this research work, unconventional PIV technique has been proposed to estimate the air entrainment in case of gas jet compared to ordinary PIV. The jet body brightness eliminates detecting the particles velocity in the detached area around the spray/jet body leading to difficulties in calculating the air entrainment into the spray or jet. Using fluorescence PIV included suspending tracer particles into the ambient to indicate velocity vector around the Methane jet. Moreover, the process of introducing the air into the high-pressure Methane jet was visualized and measured from the flow field measurement of the air flow by PIV (Particle Image Velocimetry) using a high repetition pulse laser.

Light oil JIS\#2 was injected into a visualization container with less disturbance, and the outer diameter of the spray and the flow field of the surrounding fluid was obtained. The outer shape of the spray is dark by the fluorescence PIV, making it difficult to distinguish it from the background. Midian blur and Gaussian blur were applied both before and after binarization on the image processing program to distinguish the spray that had been removed, and the tracer particles were erased to obtain the outer shape of the spray. On the other hand, the mixture entrainment process of torch flames has been discussed using the PIV technique.

\section{Experimental equipment and test procedures}

Chamber configurations of a real DF engine are hard to simulate since it requires several auxiliary PCC devices such as an ignition plug, a sub gas injector, and so on. All of them should be actuated synchronously with an engine crank angle. In the case of a constant volume vessel (CVV), the synchronization is not necessary, but the mixture control in the PCC becomes problematic because of the lack of compression and expansion strokes that assures PCC gas exchange. For overcoming the situation, rupture of a membrane was introduced in this study. The membrane 
turns the upper part of the PCC into an air pressure reservoir and low-pressure air jets eject from the nozzle holes after a solenoid-driven needle pierces the membrane for rupturing. The differential pressure between the upper chamber and the lower one was chosen as a main parameter of the experiment.

A high-speed camera Model SA-Z Monochrome (Photoron) was used for the PIV measurement with specifications shown in Table 1.

Table 1 High-speed camera specifications

\begin{tabular}{|l|l|}
\hline High-speed camera & High-speed camera \\
\hline Frame rate & $20,000 \mathrm{fps}$ \\
\hline Resolution & $1,024 \times 1,024$ pixels \\
\hline Exposure time & $23.39 \mu \mathrm{s}$ \\
\hline Lenses & Micro NIKKOR $105 \mathrm{~mm}, \mathrm{~F} 2.8+2.0$-power teleconverter \\
\hline
\end{tabular}

Table 2 High frequency light source details

\begin{tabular}{|l|l|}
\hline Nd:YAG pulse laser & LDP-100MQG (Lee Laser) \\
\hline Wavelength & $532 \mathrm{~nm}$ \\
\hline Frequency & $20 \mathrm{kHz}(\Delta t=50 \mu \mathrm{s})$ \\
\hline Pulse energy & $2.0 \mathrm{~mJ} / \mathrm{pulse}$ \\
\hline Pulse width & $4.0 \mu \mathrm{s}$ \\
\hline Timing controller & LC 880 (Labsmith) \\
\hline
\end{tabular}

The CVC has been charged with tracer particles and the suspension of the particles was insured by the aid of air pressurizing. Figure 1 illustrates the layout of the optical system which has been incorporated in the PIV technique.

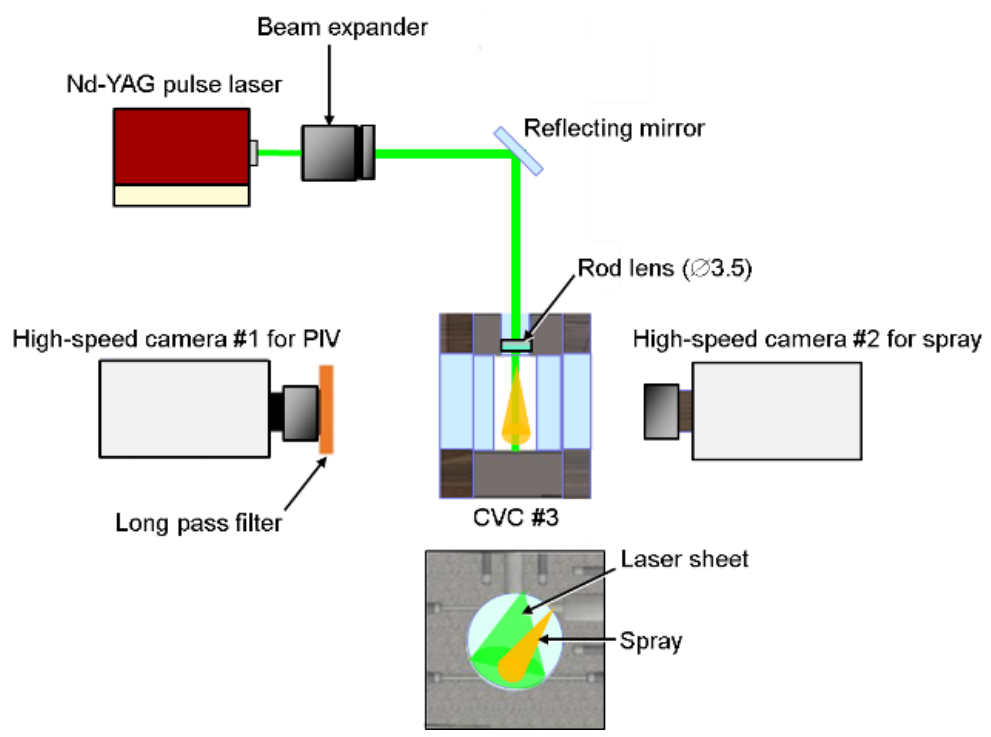

Figure 1 Fluorescence PIV optical system 


\section{Results and discussion}

In this section, the results from fluorescence PIV measurements for induced air motion around diesel sprays, a high-pressure gas jet directly injected under gas pressure as high as $30 \mathrm{MPa}$ and a directly injected medium in internal combustion engines as a torch flame ejected from nozzle holes of a pre-combustion chamber (PCC) to a main combustion chamber (MCC) of a so-called DF (dual-fuel) engine will be explained.

\subsection{Fluorescence PIV measurement principle}

In this study unconventional technique called fluorescence PIV has been employed aiming to avoid the light scattering phenomena in the conventional PIV technique as can be seen clearly from the comparison illustrated in Figure 3. Using tracer particles can enable precise and clear air entrainment calculations. To remove scattered light from the boundary surface,

Figure 2 shows SCF560 filter which has been attached to the camera lens to eliminate the laser light with $532 \mathrm{~nm}$ wavelength allowing the light discarded from the fluorescent with $590 \mathrm{~nm}$ wavelength.

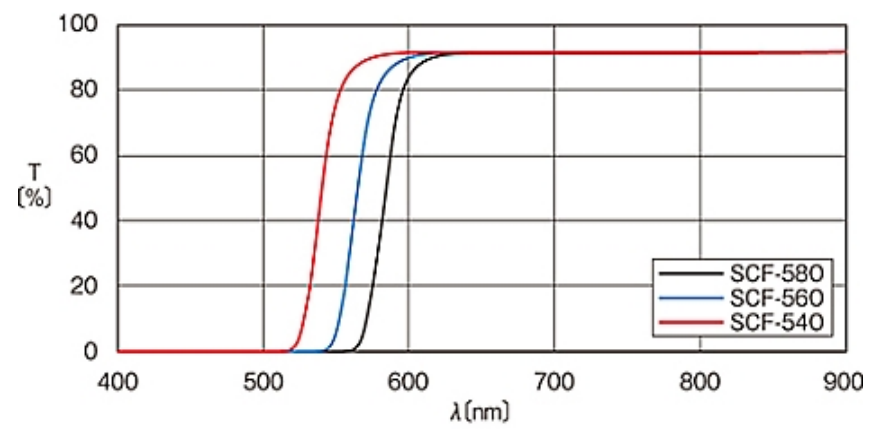

Figure 2 Frequency characteristics of sharp cut filter transmittance versus wavelength
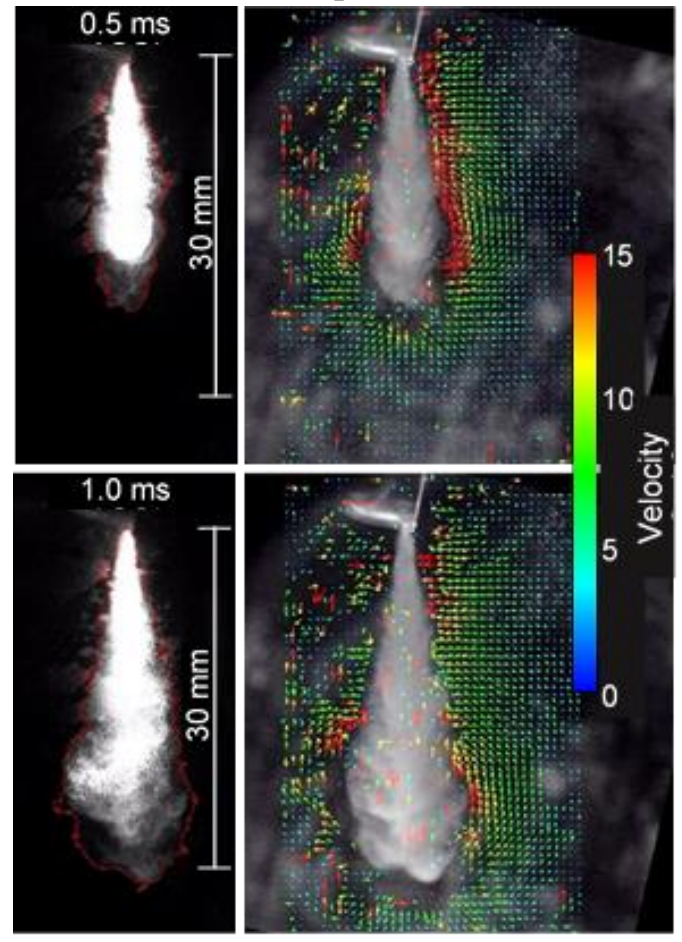

Page 4 of 10 
Figure 3 Comparison between the method in this study and the conventional method (left is conventional method while right is fluorescence PIV method)

Table 3 shows the experimental conditions in this study while Table 4 lists the details of the tracer particles used in this study.

Table 3 Experimental conditions

\begin{tabular}{|l|l|}
\hline Fuel & Light oil JIS\#2 \\
\hline Nozzle diameter & $0.125 \mathrm{~mm}$ \\
\hline Nozzles number & 6 and 8 \\
\hline Injection pressure & 60 and $70 \mathrm{MPa}$ \\
\hline Injection time & $500 \mu \mathrm{s}$ \\
\hline Ambient gas & Nitrogen \\
\hline Ambient pressure & $1.56 \mathrm{MPa}$ \\
\hline Ambient Temperature & $296 \mathrm{~K}$ \\
\hline
\end{tabular}

Table 4 tracer particles specifications

\begin{tabular}{|l|l|}
\hline Type & Godd Ball, b-5c \\
\hline Company name & SUZUKIYUSHI INDUSTRIAL CORPORATION \\
\hline Material & Hollow ball $\mathrm{SiO}_{2}$ \\
\hline Average diameter & $2-2.5 \mu \mathrm{s}$ \\
\hline Bulk Density & $180-450 \mathrm{Kg} / \mathrm{m}^{3}$ \\
\hline
\end{tabular}

Using the spray surface area calculations, Total air entrainment at two different injection pressures 60 and $70 \mathrm{MPa}$. The entrainment in case of high injection pressure seems to be accumulated after $1 \mathrm{~ms}$ ASOI while it shows more stable values in case of $60 \mathrm{MPa}$.

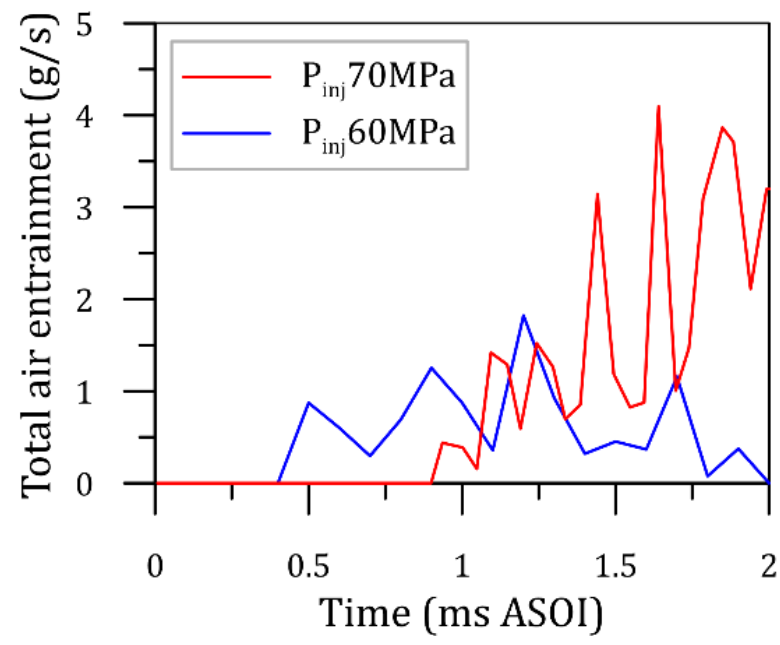

Figure 4 Air entrainment rate at $60 \mathrm{MPa}$ and $70 \mathrm{MPa}$ injection pressures

\subsection{PIV measurements for gas jet}

Table 5 PIV analysis specifications

Page 5 of 10 


\begin{tabular}{|l|l|}
\hline Software & Koncert II (Seika Corporation) \\
\hline Algorithm & Recursive direct cross-correlation method \\
\hline $\begin{array}{l}\text { Inspection window } \\
\text { size }\end{array}$ & $16^{*} 16$ pixels $(2.55 \mathrm{~mm} * 2.55 \mathrm{~mm})$ \\
\hline Overlap & $50 \%$ \\
\hline
\end{tabular}

\subsubsection{Test condition}

In the recent study, Methane gas was examined under different conditions of pressure and temperature. A 0.4-hole injector was used in the case of methane gas with rating injection pressure $(20,30$ and 40) MPa at $293 \mathrm{~K}$.

Figure 5 shows the results of PIV analysis for spray. The jet grows with a similar shape with a Capturing Section that has an exclusion flow that spreads radially at the tip of the jet and an Entrainment Section that has a flow that continues from the root and faces the inside of the jet. In addition, the flow field around the jet was found to have a size about 1.0 to $2.0[\mathrm{~m} / \mathrm{s}]$ stronger than that of spraying.

Figure 6 shows the distribution of the atmosphere introduction velocity at the spray boundary in the injection axis direction. As can be seen from the PIV results, vortices are generated actively in the jet, and an intensive air entrainment has been noticed.

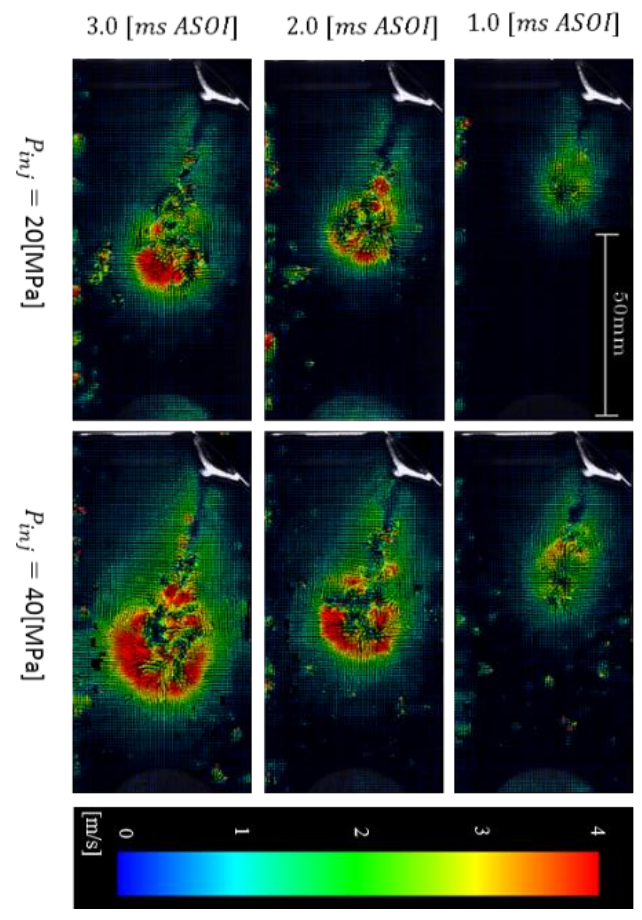

Figure 5 Flow field around Methane jet at 36.3 MPa ambient density 

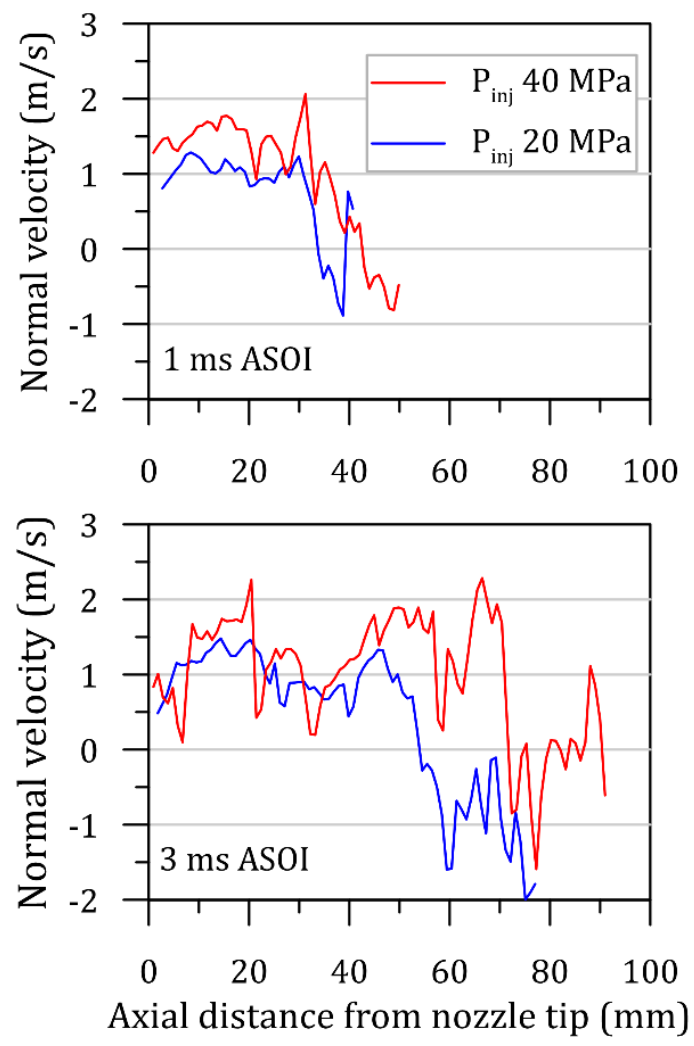

Figure 6 Distribution of accompanying flow flowing into the spray $\rho_{a}=36.3\left[\mathrm{~kg} / \mathrm{m}^{3}\right]$

Figure 7 shows the time change of the mass atmosphere introduction rate and the total introduced mass under the conditions shown in the above figure. As can be seen from the figure below, it was observed that the total mass of the ambient atmosphere introduced increased as the injection pressure increased in both cases of spraying and jetting.

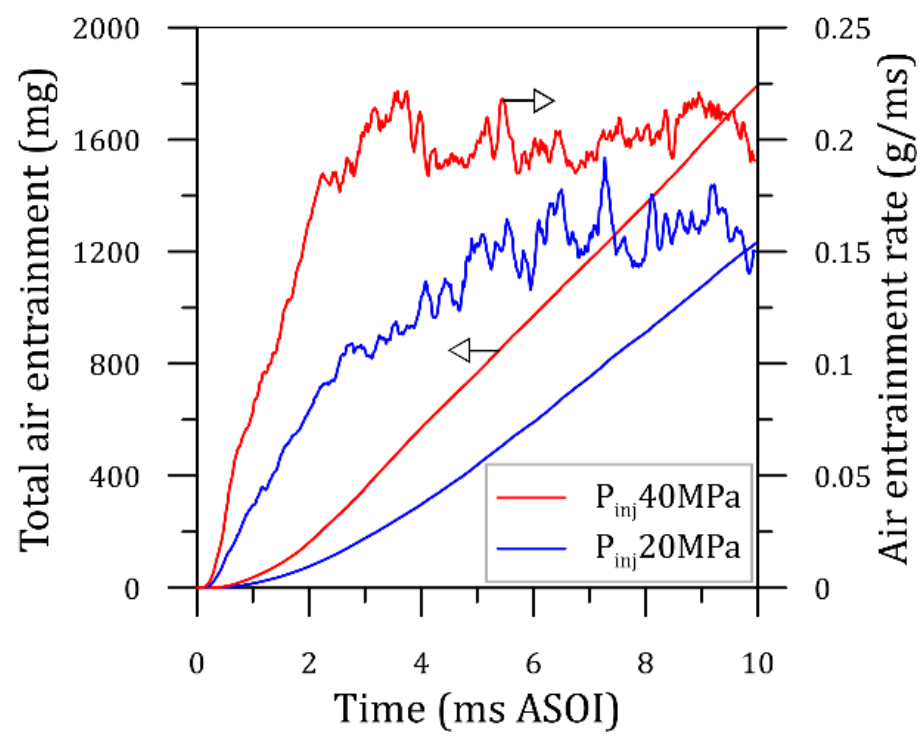

Figure 7 Air entrainment for methane jet under different injection pressures 
Air entrainment rate and total air entrainment versus time at 36.3 MPa ambient density Regarding the jet, only the introduction from the Entrainment Section is considered, but the ratio of both spray and jet is explained to be about the same, and this measurement is valid. In addition, it seems that one of the reasons for the strong introduction of jets from the Entrainment Section is the entrainment of a large atmosphere due to the large-scale vortex structure as shown in Figure 8 .

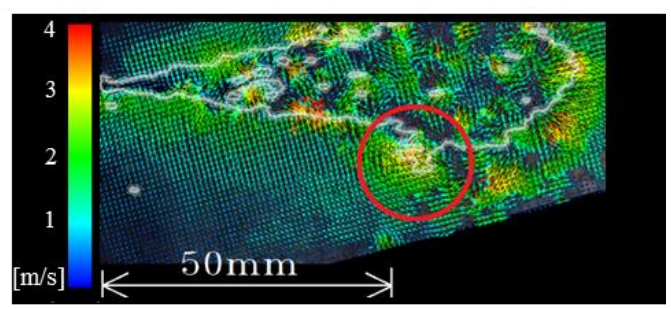

Figure 8 Distribution of the accompanying flow flowing into the spray and the moment of introduction of the atmosphere by the vortex seen in the jet $\mathrm{P}_{\mathrm{inj}}=40[\mathrm{MPa}], \rho_{a}=36.3\left[\mathrm{~kg} / \mathrm{m}^{3}\right]$

\subsection{PIV measurements for Torch flame}

\subsubsection{Test condition}

In this study, the air has been examined in the pre-chamber of the torch flame. Neglecting the amount of fuel into the pre-chamber since the fuel is lean, the air has been considered to be examined in this case. Moreover, Using the air can ensure a high level of torch flame stability.

Figure 9 illustrates the PIV measurements and spry contour for a spray in prechamber. It worth to be mention that in this case using direct shot to get the contour of the spray was not applicable due to the difficulties of density difference. Instead, the contour data has been extracted from velocity vector analysis. From the figure, it can be noticed that strong eddies occur leading to increase the air entrainment rate while no entrainment has been noticed in the capturing section due to strong pushing out. The contour of the air spray seems to be same as the ordinary shape of the diesel spray.
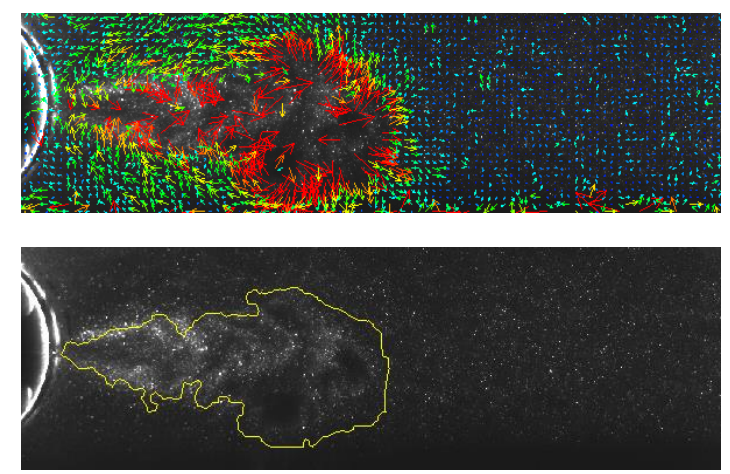

Figure 9 PIV measurements for spray in torch flame at $2 \mathrm{msASOI}$

Figure 10 shows the total air entrainment for air jet in the prechamber at different injection pressures 2, 3 and $4 \mathrm{MPa}$. It is clearly seen that the higher the injection pressure the higher the total air entrainment. This attribution could be due to the higher movements of the spray body which in turn generates more eddies allowing more air to be grasped into the main spray body. 


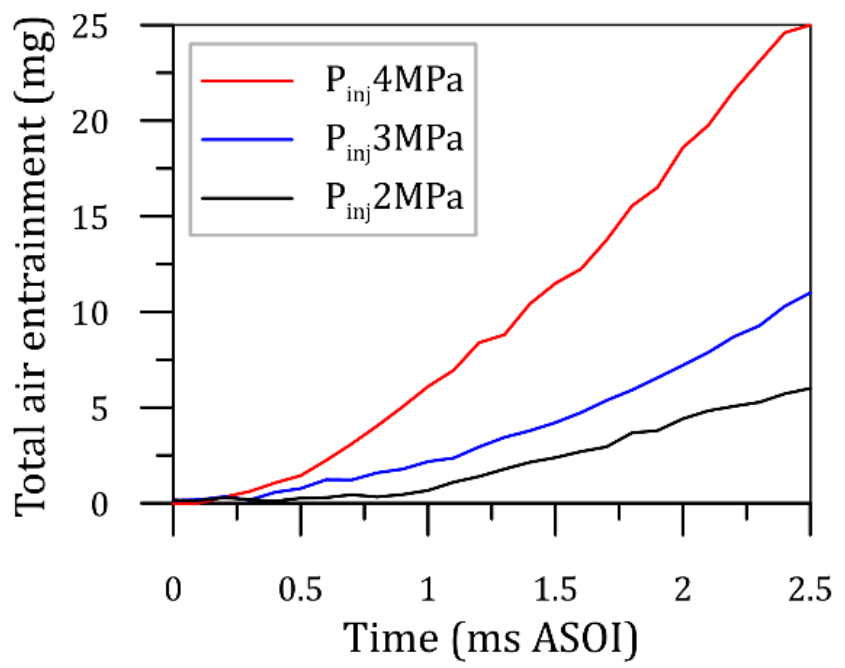

Figure 10 Total air entrainment in the torch flame

\section{Conclusion}

In this research work, florescence PIV measurements have been considered to study the entrainment process for the first time. Due to the difficulties of simulating the real DF engine, the PIV measurements have been considered as a tool for detecting the air entrainment process. The following results have been gotten: -

- The scattered light of the spray was suppressed by the fluorescence PIV, and the velocity field around the spray could be analyzed accurately.

- The air entrainment rate is directly proportional to the injection pressure.

- The high-pressure gas jet undergoes an atmosphere introduction process similar to ordinary liquid spraying, but it is observed that the atmosphere introduction in the Entrainment Section is active with a flow velocity.

\section{References}

Healy D, Curran HJ, Simmie JM, Kalitan DM, Zinner CM, Barrett AB, Petersen EL, Bourque G (2008) Methane/ethane/propane mixture oxidation at high pressures and at high, intermediate and low temperatures. Combust Flame 155: 441-448.

Lion S, Vlaskos I, Taccani R (2020) A review of emissions reduction technologies for low and medium speed marine Diesel engines and their potential for waste heat recovery. Energy Convers Manag 207: 112553.

Thiripuvanam T, Tashima H, Tsuru D (2017) Air entrainment and combustion process of highpressure gas jet in gas direct injection engines. in 9th International Conference on Modeling and Diagnostics for Advanved Engine Systems, COMODIA, Okayama, Japan, July 25-28

Xia J, Huang Z, Xu L, Ju D, Lu X (2019) Experimental study on spray and atomization characteristics under subcritical, transcritical and supercritical conditions of marine diesel engine. Energy Convers Manag 195: 958-971.

Zegers RPC, Luijten CCM, Dam NJ, De Goey LPH (2012) Pre- and post-injection flow characterization in a heavy-duty diesel engine using high-speed PIV. Exp Fluids 53: 731-

Page 9 of 10 
746.

Zhang W, Li X, Huang L, Feng M (2019) Experimental study on spray and evaporation characteristics of diesel-fueled marine engine conditions based on optical diagnostic technology. Fuel 246: 454-465.

Zhao J, Liu W, Liu Y (2020) Experimental investigation on the microscopic characteristics of underexpanded transient hydrogen jets. Int J Hydrogen Energy 45: 16865-16873. 